\title{
THE TEACHING OF ASTRONOMY IN CHINA
}

Feng Ke-Jia

Department of Astronomy, Beijing Normal University, Beijing 100875, China

\section{General Information}

Astronomical education has been developing at an increasing rate in China since 1977. Many Chinese astronomers think that the development and the popularization of astronomical education are future human needs. For this reason, we use radio and TV broadcasts as well as planetariums to popularize astronomical education in our country. The teaching of astronomy is enhanced in schools step by step. For elementary schools many astronomical topics are included in a course under the general title of Nature. Some activities such as astronomical observation and courses of astronomical lectures are organized in secondary schools. In universities, elective courses of astronomy are arranged not only in some departments of natural science but also in some departments of liberal arts. Some students in other scientific departments are encouraged to take astronomical courses, so that universities can supply frontier science with researchers.

In recent years, education in astronomy has made remarkable progress in China. Two new planetariums have been built, one in Nanjing, Jiangsu Province, and the other in Taiyuan, Shanxi Province. Two kinds of ordinary astronomical telescopes, an $80-\mathrm{mm}$ refractor and a $120-\mathrm{mm}$ reflector with equatorial mounting, both made in China, are supplied on the domestic market, which has played an important role in astronomical education. From 1984 to 1987 , about 70 different books on astronomy were published. The astronomical center for children in Suzhou, Jiangsu Province, is supplied with an equatorially mounted reflector of 400-mm aperture. The observatory in Beijing No. 4 High School houses an equatorially mounted reflector of $350-\mathrm{mm}$ aperture. These facts show the improvement in spreading astronomical knowledge.

\section{Elementary Schools}

Astronomical education at the elementary level is included in a course under the general title of Nature. From grade four to grade six, some general knowledge of astronomy is introduced. In grade four, for instance, pupils are required to recognize constellations, to use sundials, and to determine the solar altitude. In grade five, pupils are taught movement of constellations in the four seasons. In grade six, pupils are introduced to the moon, solar eclipses, lunar eclipses, the solar system, the Milky Way, galaxies, and the universe in general. Sometimes pupils may do some observations, or visit astronomical clubs. It is expected that some elementary schools will set up their own observatories with small telescopes. 


\section{Secondary Schools}

Astronomical education at the secondary level is included in courses under the general titles of Geography and Physics. At the senior high level, chapters on the Earth and the universe are incorporated in Geography. In the chapter on the celestial bodies, students are required to learn about the celestial sphere, stars, and nebulae. In the chapter on the sun, students are required to learn some knowledge of the structure of the solar atmosphere, solar activity, solar-terrestrial relationships, and the region of solar nuclear reactions. In the chapter on the solar system, students are initiated into the movement and structural characteristics of planets, the phases of the moon, and the Earth-moon system. In the chapter on movement of the Earth, students are introduced to the rotation of the Earth, the axis of rotation, the obliquity of the ecliptic, and seasonal variation.

There is a chapter on gravitation in the course of Physics at the senior-high level. As a matter of course, planetary motion, the law of gravitation, and artificial satellites are introduced in this chapter. It is expected that astronomy will be given a greater share at the senior-high level in future.

\section{University Education}

At present there are four major facilities for education in astronomy: the Department of Astronomy at Nanjing University, the Department of Astronomy at Beijing Normal University, the Speciality of Astronomy at Beijing University, and the Center for Astrophysics at the University of Science and Technology of China.

Since 1984 about 135-140 undergraduates and 55-60 graduates have graduated from the four major facilities.

In China, other universities offer astrophysics and/or general astronomy as elective courses. Usually, astrophysics is taught in departments of physics, and general astronomy is taught in departments of geography, departments of philosophy, and other departments. It is anticipated that more and more universities will introduce these programs.

The length of the degree course is four years for undergraduates in the astronomy departments and there are many resemblances among the courses at other universities.

There are three principal kinds of courses in an astronomy department. They are the obligatory course, the restricted elective course, and the elective course. The undergraduates in the astronomy department must learn many kinds of obligatory courses, such as foreign languages, mathematical analysis, differential equations, linear algebra, general physics, experimental physics, methods of mathematics and physics, theoretical mechanics, fundamental astronomy, etc. Those courses are arranged in the freshman and sophomore years (first and second years). There are three kinds of restricted elective courses; astrophysics, astrometry, and celestial mechanics are offered in the junior and senior years (third and fourth years) in the astronomy department. The following restricted elective courses in astrophysics can be selected by undergraduates specializing in astrophysics: fiuid mechanics, electro- 
dynamics, practical astrophysics, methods of radio astronomy, thermodynamics and statistical physics, etc. The following restricted elective courses of astrometry can be selected by undergraduates specializing in astronomy or astrometry: methods of data processing, theory of probability and data processing, measurement of the rotation of the Earth, theory of the rotation of the Earth, star catalogues and astronomical constants, fundamentals of celestial mechanics, etc. The following restricted elective courses of celestial mechanics can be selected by undergraduates specializing in celestial mechanics: functions of real variables, elementary topology, advanced differential equations, celestial mechanics, methods of celestial mechanics, theory of the orbits of artificial satellites, quantitative theory of celestial mechanics, etc. By the way, the undergraduate can also select three or more of the following topics of the special elective courses: plasma physics, theory of stellar atmospheres, solar physics, radio astrophysics, solar-terrestrial physics, stellar physics, astrophysics, cosmical physics, etc.

\section{Public Education}

There are four planetariums in China. A number of astronomical clubs can be found all over the country. These clubs usually are connected with the local Association of Science and Technology.

The Chinese Astronomical Society has always paid great attention to bringing up young amateur astronomers. These young astronomical enthusiasts pay regular visits to observatories and planetariums and are directly trained by astronomers there. For instance they observed Halley's Comet in 1986 and the annular solar eclipse on September 23, 1987.

The researchers of planetariums and clubs help to encourage public interest in astronomy and to provide the newspapers, radio, and TV with necessary astronomical information.

\section{References}

(1) Amateur Astronomer, Published Monthly by Amateur Astronomer Publishing House, Beijing Planetarium, Beijing, China, Nos. 122-158.

(2) "The Present State of Astronomical Education in China" by Feng Ke-Jia, Supplement to Proceedings of the Third Asian - Pacific Regional Meeting of the International Astronomical Union, Sept. 30-Oct. 6, 1984, Kyoto, Japan, pp $18-22$. 